\title{
User-Centered Design of a Dynamic-Autonomy Remote Interaction Concept for Manipulation-Capable Robots to Assist Elderly People in the Home
}

\author{
Marcus Mast, ${ }^{1}$ Michael Burmester, ${ }^{1}$ Katja Krüger, ${ }^{1}$ \\ Sascha Fatikow, ${ }^{1}$ Georg Arbeiter, ${ }^{2}$ Birgit Graf, ${ }^{2}$ Gernot \\ Kronreif, ${ }^{3}$ Lucia Pigini, ${ }^{4}$ David Facal, ${ }^{5}$ and Renxi Qiu ${ }^{6}$ \\ ${ }^{1}$ Stuttgart Media University, Germany \\ ${ }^{2}$ Fraunhofer Institute for Manufacturing Engineering and \\ Automation (IPA), Germany \\ ${ }^{3}$ Austrian Center for Medical Innovation and Technology \\ (ACMIT), Austria \\ ${ }^{4}$ Fondazione Don Carlo Gnocchi, Italy \\ ${ }^{5}$ Instituto Gerontológico Matia (Ingema), Spain \\ ${ }^{6}$ Cardiff University, United Kingdom
}

In this article, we describe the development of a human-robot interaction concept for service robots to assist elderly people in the home with physical tasks. Our approach is based on the insight that robots are not yet able to handle all tasks autonomously with sufficient reliability in the complex and heterogeneous environments of private homes. We therefore employ remote human operators to assist on tasks a robot cannot handle completely autonomously. Our development methodology was user-centric and iterative, with six user studies carried out at various stages involving a total of 241 participants. The concept is under implementation on the Care-O-bot 3 robotic platform. The main contributions of this article are (1) the results of a survey in form of a ranking of the demands of elderly people and informal caregivers for a range of 25 robot services, (2) the results of an ethnography investigating the suitability of emergency teleassistance and telemedical centers for incorporating robotic teleassistance, and (3) a uservalidated human-robot interaction concept with three user roles and corresponding three user interfaces designed as a solution to the problem of engineering reliable service robots for home environments.

Keywords: Human-robot interaction, dynamic autonomy, semi-autonomous robots, teleoperation, elderly people, interaction design, user interfaces

\section{Introduction}

People prefer to grow old in the familiar environment of their home rather than in care facilities (Bayer \& Harper, 2000). However, declining physical or mental abilities often lead to dependence on caregivers or institutionalization. Assistive robots have the potential to retain or restore elderly people's independence in the future. Contrary to frequently expressed opinion, most elderly

Authors retain copyright and grant the Journal of Human-Robot Interaction right of first publication with the work simultaneously licensed under a Creative Commons Attribution License that allows others to share the work with an acknowledgement of the work's authorship and initial publication in this journal. 
people are open to the idea of a robot assisting them at home (Ezer, Fisk, \& Rogers, 2009; Mast et al., 2010; Meyer, 2011).

Motivated by the demographic trend of population aging (United Nations, 2010), many robotics projects have emerged with the aim of assisting elderly people. Until recently, most robots in this field were restricted to non-physical tasks like providing information (Pollack et al., 2002), telepresence (Michaud et al., 2010; Beer \& Takayama, 2011), or emotional stimulation (Wada et al., 2007). The long-term vision is for robots to also assist physically, helping with everyday chores that have become difficult or impossible to accomplish. Providing assistance with physical tasks requires robots with manipulation capabilities. Examples of domestic service robots with manipulation capability are PR2 (Bohren et al., 2011), Twendy-One (Iwata \& Sugano, 2009), and Care-O-bot 3 (Graf, Parlitz, \& Hägele, 2009).

For robots, acting autonomously in a private home is immensely challenging. The environment is unstructured, built for humans, highly heterogeneous, and dynamically changing. There are obstacles like chairs or doors, human beings can be in proximity of the robot, and there is a multitude of tasks to cover. This leads to challenges in nearly all technological areas, such as perception and sensing in 2D and 3D, safe and successful navigation and manipulation, detection and interpretation of human behavior, and acquisition of new skills and knowledge (Kemp, Edsinger, \& Torres-Jara, 2007; Goodrich \& Schultz, 2007). The challenges have led to the development of highly specialized robots that can only cover one or a few tasks like vacuuming, lawn mowing, or emotional stimulation (e.g., Wada et al., 2007). However, to profoundly support people in their daily life, sophisticated multi-purpose robots are required that can cover a wide spectrum of tasks. Such robots are also anticipated by people (Sung, Christensen, \& Grinter, 2009). While many research institutes are working towards such robots, no products able to meet the high expectations have come to the market yet.

An alternative to autonomous robots are teleoperated robots (Sheridan, 1992; Beer \& Takayama, 2011; Weiss et al., 2009). Conventional low-level teleoperation (e.g., manually steering the robot with a joystick) requires no or very little robotic autonomy, eliminating many of the above-mentioned problems as they are related to the robot's own sensing and decision making. There is a wide variety of possible interaction devices such as joysticks, accelerometer-based devices (Song et al., 2007), data gloves, force feedback devices (O'Malley \& Ambrose, 2003), and touch screens (Weiss et al., 2009). Teleoperation can benefit from using augmented displays (Nielsen, Goodrich, \& Ricks, 2007; Michaud et al., 2010), sensor fusion (Ferland et al., 2009, Nielsen et al., 2007) or three-dimensional, exocentric visualizations (Nielsen et al., 2007; Michaud et al., 2010, Ferland et al., 2009) to improve situation awareness (Endsley, 1988) and telepresence (Sheridan, 1992). However, teleoperation is also associated with shortcomings. Technological challenges include network latencies, bandwidth limitations, or the restricted field of view of cameras, which can result in suboptimal operation experiences. Telemanipulation usually requires sophisticated and complex interaction devices (e.g., O'Malley \& Ambrose, 2003; Buss et al., 2010). In the context of assisting elderly people, the most severe limitation however seems to be the constant need for a human operator. When considering elderly end-users as operators, the required interactions may be too difficult to handle (Labonté, Boissy, \& Michaud, 2010). When considering other people as operators, the concept of a service robot can be questioned, as it should free human capacities and support independent living of elderly people.

An approach that seeks to combine the advantages of autonomous and teleoperated robots is semi-autonomy. On the one hand, teleoperation can be supported by sensor-based control functions, e.g. to lead a robot's gripper along the correct path when turning handwheels remotely (Arbeiter et al., 2009). On the other hand, a robot can act autonomously to the degree that is within its capabilities but request human involvement if a situation cannot be handled autonomously with the desirable robustness. Shiomi et al. (2008) implemented a semi-autonomous control architecture to compensate for shortcomings of the robot's voice recognition. The robot approached people autonomously and started a conversation. It requested a human operator to take 
over the conversation when it detected problems in the communication. Martens, Prenzel, and Gräser (2007) employed user input to identify objects that should be grasped in a camera image.

The notion of dynamic or adjustable autonomy (Schermerhorn \& Scheutz, 2009) takes the idea of semi-autonomy a step further by integrating autonomy, semi-autonomy, and low-level teleoperation into a unified control scheme where the human operator or the robot can switch between control modes of varying autonomy. Bruemmer, Dudenhoeffer, and Marble (2002) implemented four control modes for navigating a robot, ranging from full teleoperation to full autonomy. Dorais et al. (1998) used the approach in various space machines where human operators took control when unexpected situations occurred. Crandall and Goodrich (2001) applied the concept for enabling a single human operator to control multiple robots simultaneously.

It can be advantageous to combine semi-autonomous or dynamic-autonomy control schemes with human teaching or machine learning (Thomaz \& Breazeal, 2008; Nehaniv \& Dautenhahn, 2007). This way, a robotic system can evolve its capabilities by learning from human input, becoming more autonomous over time, extending its functional range, and adapting to unknown environments (Mason \& Lopes, 2011; Bruemmer et al., 2002).

We aim to make physically assistive multi-functional service robots for elderly people more feasible in the near term. Autonomous robots are unlikely to achieve success rates close to $100 \%$ for a wide range of tasks for some time and exclusive teleoperation fails to free human resources. Thus, we regard semi-autonomy and dynamic autonomy combined with learning systems a promising approach. The present article describes a user-validated interaction concept for realizing such an approach.

\section{Research Procedure}

Our overall goal was to obtain a well-founded, user-validated interaction concept for realizing the idea of a dynamically autonomous robot acting in the home of an elderly person, supported by remote operators for tasks that cannot be executed autonomously or fail during execution, with the robot learning from user input. The concept was to be applicable to most of today's mobile, manipulation-capable service robots intended for home use. We applied a methodology with extensive involvement of users, in accordance with the philosophy of user-centered design (ISO 9241-210, 2010), to gradually improve and validate our ideas. Altogether, we carried out six user studies at various stages of the project, involving a total of 241 participants in three European countries.

Figure 1 illustrates the research procedure followed. Two main phases can be distinguished: preparatory studies (user studies 1 to 4 and analytical studies 5 to 7 ) and the iterative conceptual design (segments 8 to 12). The present article focuses on two preparatory studies (segments 2 and 4; Section 3) and the human-robot interaction concept along with its user interface designs (segment 12; Section 4). To provide the overall context of the actions carried out, in this section, we describe the methods applied and some brief results of the studies not within the focus of this article.

Focus Groups (Segment 1). We started with the basic idea of a robot in the home of an elderly person, which would be assisted remotely (e.g., by family or professional staff). Illustrations and explications of how this could take place in everyday life were discussed with potential users in a focus group study (Merton, Fiske, \& Kendall, 1990). The study also investigated difficulties of elderly people and caregivers to be considered in the conceptual design and collected ideas for possible robot services. The focus groups involved a total of 59 participants (elderly people between 65 and 90, experiencing difficulties in instrumental activities of daily living, as well as caregivers) in three countries (Germany, Italy, Spain). Results have been reported in Mast et al. (2010) and some results are mentioned in Section 4.1. Users were generally supportive of the concept but also mentioned important factors to consider such as time constraints of informal (family, friends) caregivers. 
Mast et al., Remote Interaction Concept for Assistive Robots

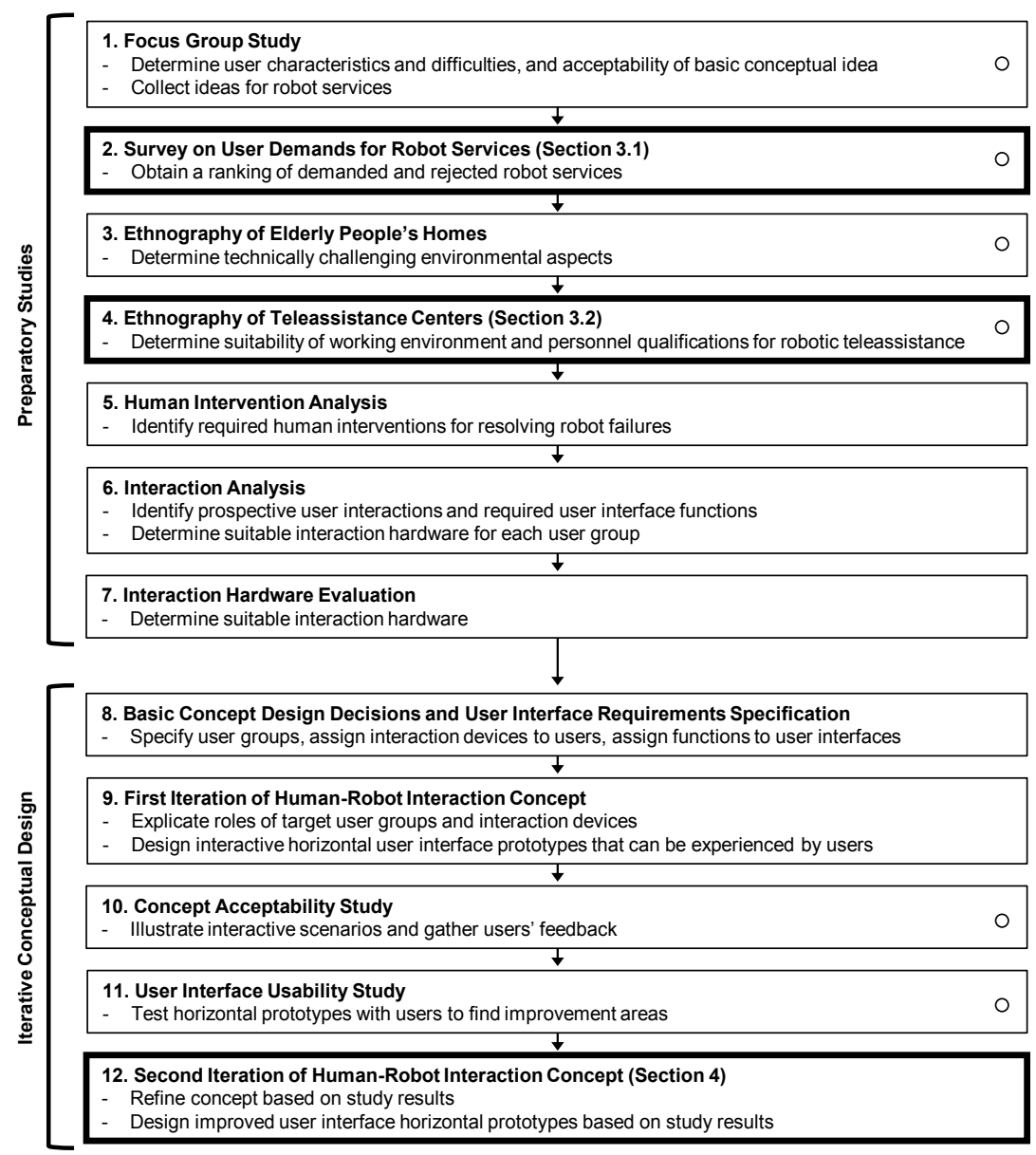

Figure 1. Procedure followed for developing the interaction concept (circles denote segments with user involvement; highlighted segments are focused in this article)

Survey on User Demands for Robot Services (Segment 2). We subsequently quantified user demands for a range of possible robot services elicited in the focus groups using a questionnaire. This survey is described in Section 3.1.

Ethnography of Elderly People's Homes (Segment 3). The main purpose of this study segment was to determine challenging environmental aspects in an elderly person's home for a service robot. We carried out an ethnographic study (Courage \& Baxter, 2005) in 15 elderly people's homes (10 in Spain, 5 in Italy), employing participant observation, diaries of daily routines, photographs, and interviews. Some of the results have been reported in Zamora et al. (2011). Determined technical challenges include narrow passages, carpets, high doorsills, delicate items like ceramics, difficult illumination conditions, and cramped spaces. The results informed technical developers and an interaction analysis (segment 6).

Ethnography of Teleassistance Centers (Segment 4). We carried out a second ethnography to determine whether current teleassistant centers for remotely supporting elderly people would be suitable for additionally offering remote assistance through a robot. This study is described in Section 3.2.

Human Intervention Analysis (Segment 5). Since our concept foresees that human operators assist when robotic autonomous task execution fails, it was important to understand what kind of tasks users would have to carry out. We therefore segmented robotic task execution and identified 
pre-conditions and post-conditions for sequential actions (e.g., a pre-condition for the action of grasping is that the arm has reached the pre-grasp position). Not meeting a condition was defined as a failure. A human intervention would then be needed to resolve the failure state. We identified 28 different human interventions. Examples of determined failure-intervention pairs are: (a) failing to plan a trajectory - human modification of the trajectory, manual navigation, or specifying a non-passable region; (b) failing to recognize an object - human reduction of search space or teaching of object. Results informed segments 6 and 7 .

Interaction Analysis (Segment 6). While in segment 5 we determined what a user would have to do to resolve a failure state, we subsequently investigated how this could be done in the form of interactions on eight candidate devices. Also, we determined additional interactions, not directly related to resolving failure states. Using the structured design method of scenario-based design (Rosson \& Carroll, 2008), we generated 21 comprehensive user interaction scenarios. We then isolated and consolidated the interactions contained, resulting in a list of 84 required user interactions. Examples of isolated interactions are: (a) to initiate a robot service as local elderly user (also when located in a different room); (b) to specify a gripper target position in a 3D scene of the environment. We used the results for identifying appropriate interaction devices (segment 7) and for specifying user interface requirements (segment 8).

Interaction Hardware Evaluation (Segment 7). To determine the most appropriate interaction hardware, we evaluated eight commercially available solutions, including a traditional PC, a solution with a controller-free gesture recognition device, mobile touchscreen devices, and various professional 3D interaction devices. Two user interface design experts and two roboticists rated suitability of the solutions for each of the 84 determined interactions. A summary of strengths and weaknesses was created for each solution. We could not identify a single hardware solution that would adequately support all required interactions. We thus specified different hardware for different user groups in the interaction concept (see Section 4.4).

Basic Concept Design Decisions and UI Requirements Specification (Segment 8). Based on the results of the preparatory studies and on conceptual goals, we made concept decisions and specified user interface requirements (see Section 4.1 for examples).

First Iteration of Human-Robot Interaction Concept (Segment 9). The knowledge gained on users and technological aspects in the preparatory stage and the specified requirements were the basis for developing a human-robot interaction concept with three user groups (elderly people, informal caregivers, professional teleassistants) with specific roles and interaction hardware. For each user group, we developed an interactive horizontal user interface prototype.

Concept Acceptability Study (Segment 10). After the initial concept had been generated, we described and illustrated usage situations where the three user groups operated the envisioned interaction devices in everyday life to control robots. 30 elderly people over 65 (mean age 83), 23 informal caregivers, and 5 professional teleassistants were interviewed about their perceptions. Results showed that the conceptual parameters were acceptable. For example, compared to the presentation of the concept in the focus group study (segment 1), concerns raised by family caregivers about increased burden because of constant availability with such a system no longer persisted as the concept now included professional teleassistants available 24 hours a day.

User Interface Usability Study (Segment 11). To improve the first iterations of the user interface prototypes, we carried out a usability study (Dumas \& Fox, 2008). The three user interface prototypes were tested with representative users using the methods of thinking aloud, observation of behavior, interviews, and the AttrakDiff questionnaire (Hassenzahl, Burmester, \& Koller, 2003). Five elderly users, seven informal caregivers, and seven professional teleassistants worked on realistic tasks in sessions of approximately 60 minutes each. Overall, we found 63 usability problems, leading to 41 design changes for the second iteration. Some of the results are mentioned in the description of the user interfaces in Section 4.

Second Iteration of Human-Robot Interaction Concept (Segment 12). Based on the results of the user studies of segments 9 and 10, we generated a revised human-robot interaction concept and 
revised user interface prototypes. The revised concept and user interfaces are the main subject of the present article, described in Section 4.

\section{Two Selected Preparatory User Studies}

This section describes two of the preparatory studies carried out for developing the interaction concept: a questionnaire-based quantification of user demands for a range of robot services and an ethnography of teleassistance centers to support elderly people living at home.

\subsection{Survey of User Demands for Robot Services}

Perceived usefulness is an important factor for the acceptance of technology (Davis, 1989; Gonzalez et al., 2011; Deegan et al., 2008). Users want to gain significant personal benefits from a robot (Ezer et al., 2009). While people generally anticipate universal robots that can cover a wide range of services (Sung et al., 2009), certain services will usually have to be prioritized in development. To support design and implementation decisions, we were interested in quantified demands of elderly people and informal caregivers for possible robot services in the home. Quantified demands for robot services have previously been produced by Khan (1998), Dautenhahn et al. (2005), Harmo et al. (2005), Ray, Mondada, and Siegwart (2008), Ezer et al. (2009), and Faucounau et al. (2009) but with other user groups, in contexts other than elderly care at home, or for smaller ranges of investigated services.

\subsubsection{Method}

In a preceding focus group study (Mast et al., 2010) we collected a range of 27 possible robot services. A supplementary literature review (Khan, 1998; Dautenhahn et al., 2005; Harmo et al., 2005; Becker et al., 2007; Boissy et al., 2007; Ray et al., 2008; Faucounau et al., 2009) yielded another seven. We consolidated the resulting 34 robot services to 25 by joining similar ones and omitting services that required a robot that can leave the house (e.g., taking out the garbage, going shopping) or that we considered unfeasible with currently available general-purpose domestic service robot hardware (e.g., lifting people requires very specialized robots able to carry heavy weight; Mukai et al., 2010).

We generated a questionnaire, which first explained our concept of a robot that would sometimes be remotely assisted by family members or professional operators and then the 25 robot services, supported by illustrations and still pictures of videos (Figure 2). The questionnaire asked to rate the usefulness of each service considering the respondent's current care situation on a five-point scale from "not useful at all" $(-2)$ to "very useful" $(+2)$ with a neutral middle. We recruited 83 participants from social meetings and support groups for elderly people and informal caregivers in Germany (38 participants), Italy (19 participants), and Spain (26 participants). We did not mention a robot-centered subject during recruitment. Questionnaires were completed in single interviews by 64 elderly people (64\% female, age 65 to 92 , mean age 77) living at home and experiencing difficulties with instrumental activities of daily living (IADL; cf. McDowell, 2006) and by 19 informal caregivers (88\% female, age 29 to 69 , mean age 54).

\subsubsection{Results and Discussion}

The survey results (Figure 3) show substantial differences between robot services in their rated usefulness. For many items, participants' responses were heterogeneous, which is reflected in the large confidence intervals in Figure 3. Nevertheless, mean ratings can give an indication of the most widely accepted and rejected services. Analyses of variance showed that the differences between mean item ratings are statistically significant for both, elderly people $(\mathrm{p}<0.0005)$ and informal caregivers $(\mathrm{p}<0.0005)$. 
Mast et al., Remote Interaction Concept for Assistive Robots


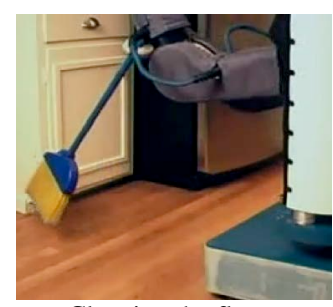

Cleaning the floor

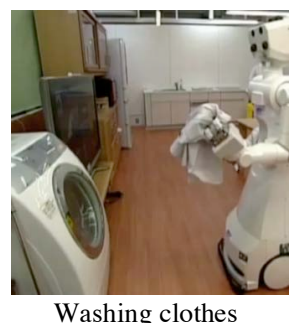

Washing clothes

Figure 2. Examples of illustrations and pictures used in the questionnaire for explaining concept (top) and robot services (bottom)

Elderly people rated emergency assistance as most useful on average (+1.3). Also, many physically strenuous housekeeping and mobility-related tasks were rated highly by elderly people (e.g., items $2,4,5,7,9,10$ ). Only 5 of the 25 items received negative mean scores (items 21 to 25), with two items with social, interactive, and emotional aspects marking the lower end: playing games with relatives through the robot (item 24: -0.3) and companionship by the robot (item 25: 0.4). Ezer et al. (2009), with a sample skewed towards elderly people but also containing younger people, investigated preferences for three groups of robot services. The group focusing on emergency and safety-related services was rated highest, the group focusing on physical housekeeping tasks was rated in the middle, and the group focusing on social tasks was rated the lowest. Although not exclusively based on elderly people's responses, these results are principally compatible with ours.

Informal caregivers rated items related to reminder functions (item $6:+1.8$; item 11: +1.8 ) and emergency (item 1: +1.7) most highly. These results are in agreement with a study by Faucounau et al. (2009) where cognitive stimulation, fall detection, and help calls were rated most highly by informal caregivers. Also, an appointment reminder function scored fairly well in their study. However, their study focused on services to address cognitive impairments rather than querying services from a broader range of areas as in the present study. Walking assistance received the only negative mean score from caregivers (item 18: -0.3) in our study and the companionship function the only neutral mean score (item 25: 0.0).

Examining differences between the ratings of elderly people and caregivers, per-item independent-samples t-tests revealed statistically significant differences at the 0.05 level between mean scores for 9 items. Elderly people do not seem to find reminder functions as useful as caregivers. While, as mentioned above, the two reminder items 6 and 11 were the most highly rated ones by caregivers, close to "very useful" $(+1.8 ;+1.8)$, they were only rated "useful" by elderly people $(+0.8 ;+0.9)$. Significant differences were further found for items 7 (opening containers), 12 (helping with electronics), 14 (doing the laundry), 16 (video conversation with relative), 19 (tidy up room), 21 (clearing table), and 24 (playing social games). For all these items, caregiver scores were substantially higher. The different results may be due to elderly people being less inclined to find help by a robot useful for the respective underlying difficulties. Alternatively, the different results may be due to different assessments of the severity of the elderly people's underlying difficulties and their general need of help by the elderly people versus by caregivers. Further, we did not control for underlying difficulties of the elderly sample versus the elderly persons assisted in the caregiver sample, so differences in the elderly people's actual 
Mast et al., Remote Interaction Concept for Assistive Robots

1. "Emergency": Assistance in case of emergency, e.g. after falling (emergency call, remote help)

2. "Reach objects": Robot fetches objects difficult to reach (e.g. high on shelf or on the floor)

3. "Reading": Robot reads out aloud small letters on food packages, medicine leaflets, books, etc.

4. "Floor": Robot wipes and vacuums floor

5. "Windows": Robot cleans windows

6. "Appointment reminder": Robot reminds of appointments, times for activities, etc.

7. "Containers": Robot opens containers like food cans,

8. "Purchases": delivery service brings shopped food; robot opens door, accepts, places purchases in fridge, etc.

9. "Fetch and carry": Robot fetches objects (e.g., a drink

10. "Heavy objects": Robot fetches and carries heavy

11. "Medication reminder": Robot reminds to take medication (pills, eye drops, etc.)

12. "Electronics": Robot helps operating electronic devices like TV (e.g., with help of remote operator)

13. "Rising": Robot helps to rise from chair or bed, e.g., by offering an arm

14. "Laundry": Robot does the laundry, hangs, folds, puts away clothes

15. "Bathtub": Robot helps to climb in or out of bathtub or

16. "Video call": Robot establishes video call with family or friends

17. "Dishwasher": Robot loads and unloads dishwasher

18. "Support walking": robot supports walking by escorting person and offering arm

19. "Tidy up room": Robot brings objects in the apartment back to where they belong

20. "Dressing": Robot helps with dressing

21. "Table": Robot clears away things on table (e.g., after a meal)

22. "Body": Robot helps with bathing and body washing

23. "Cooking": Robot cooks or warms up meals

24. "Games": Robot initiates remote session to play board games (e.g., chess) with relative or friend

25. "Companion": Robot talks with elderly person and provides companionship

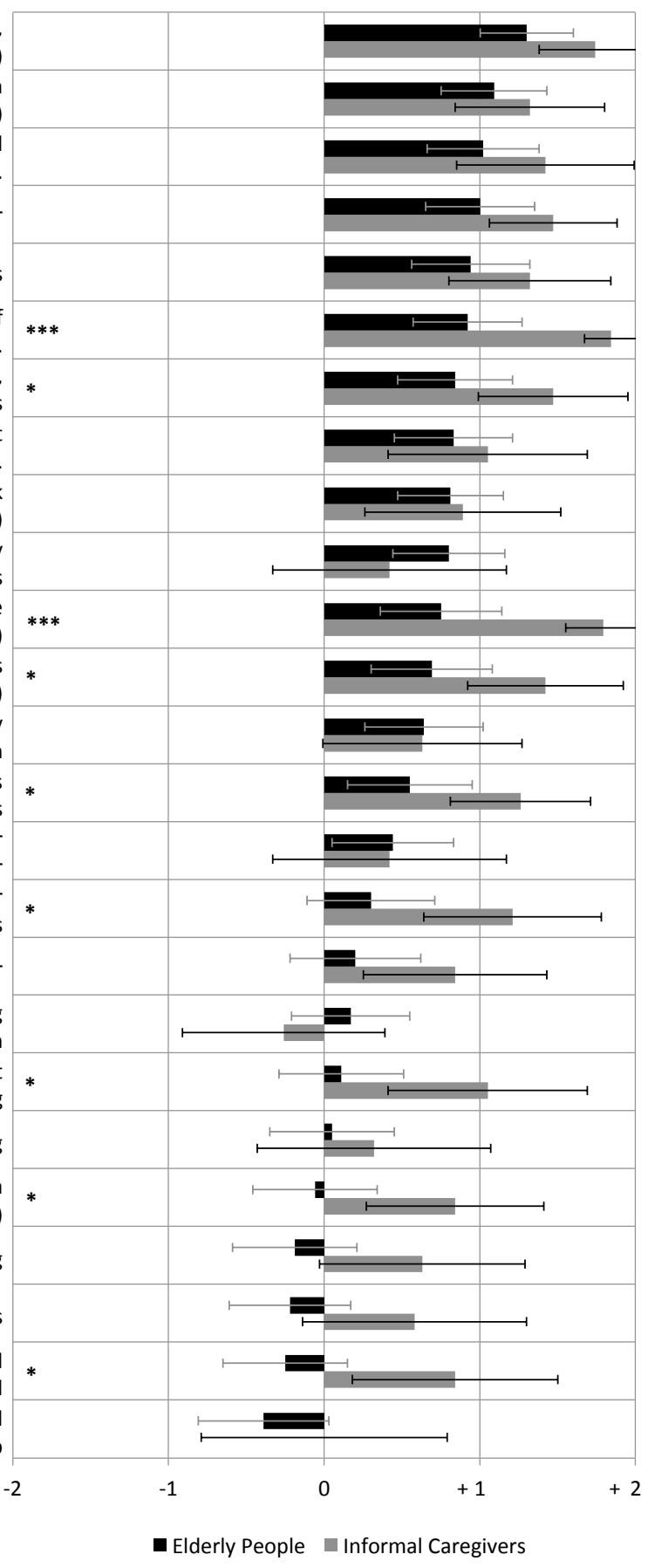

- Elderly People Informal Caregivers

Figure 3. Robot services demanded and rejected by elderly people and informal caregivers (means and $95 \%$ confidence intervals), sorted by demand of elderly people; 5-point rating scale from "not useful at all" (-2) to "very useful" (+2); asterisks denote statistically significant differences between mean ratings of user groups (* at 0.05 level, $* * *$ at 0.001 level) 
underlying difficulties may also be an explanation. Ezer et al. (2009) compared three groups of robot services (emergency and safety, housekeeping, social tasks) between older and younger adults (but not specifically caregivers). Only for the group containing on emergency and safety services they found statistically significant differences between older and younger adults. In their study, however, older adults rated those services higher than younger adults. While the two studies are not directly comparable, it would be interesting to investigate the reasons for these different results in the future.

Overall, most mean ratings are on the positive side of the scale, indicating fairly high acceptance of most services by both user groups. High acceptance of robot services has previously been found for elderly people (Ezer et al., 2009; Meyer, 2011) and informal caregivers (Faucounau et al., 2009). However, none of these studies directly compared ratings between elderly people and informal caregivers. As we had the impression that informal caregivers generally rated higher than elderly people, we carried out a post-hoc analysis showing that the average per-person mean rating was +1.0 for caregivers versus +0.5 for elderly people. An independent-samples t-test showed this difference to be statistically significant $(\mathrm{p}=0.026)$. The higher overall rating by caregivers in our study may indicate higher perceived usefulness and acceptability of an assistive robot. Perhaps the caregivers saw stronger benefits for themselves due to an ease of their own burden (cf., Mast et al., 2010), or, due to higher experience with technology, they may have been more inclined to believe that a robot could really benefit the life of their assisted person. It is however also conceivable that the reasons outlined in the previous paragraph - different judgments of the severity of underlying difficulties and different actual underlying difficulties - contributed to this result. These questions would have to be clarified by further research.

\subsection{Ethnographic Study of Teleassistance Centers}

Our focus group study (segment 1 in Section 2; Mast et al., 2010) showed that informal caregivers have significant time restrictions as they are often at work and thus would not always be available as remote operators. Also, they were often afraid of an increased burden of availability, for example being contacted for remote assistance by an elderly person when on vacation. Further, our interaction analysis (segment 6 in Section 2) showed that some required interactions would likely be too complex to handle for untrained users. Therefore, we considered professional operators a potentially necessary user group for remotely assisting a robot according to the investigated concept. However, a profession as remote operator of a care-related service robot does not yet exist. We considered the closest match to be current teleassistance personnel remotely supporting elderly people calling through emergency alerting systems.

We wanted to know if the working environment and staff in such institutions could be suitable for the task of assisting remotely with a robot. Our goals in this study were to learn about working environments, competences, tasks and routines, and artifacts in use. While there are published field studies in areas like elderly home telecare (Milligan, Roberts, \& Mort, 2011) and telehealth (Hibbert et al., 2004), we are not aware of studies with a focus similar to ours.

\subsubsection{Method}

We carried out an ethnographic study in four institutions in Germany dealing with the needs of elderly people: three home emergency teleassistance centers and one telemedical institution. We interviewed five participants (all female, age 26 to 43, mean age 33) using contextual inquiry (Beyer \& Holtzblatt, 1998) in sessions of approximately two hours each. With the contextual inquiry method, the researcher adopts the role of an apprentice and the participant teaches him his or her work. We made audio recordings of the interviews, took photos of the work environment and artifacts in use, and notes. From the audio recordings we generated transcripts. Based on the collected data, we produced affinity diagrams of participant statements to identify common themes, 
sequence models of tasks carried out, physical models (sketches) of the work environments, and personas (Beyer \& Holtzblatt, 1998; Cooper, 1999).

\subsubsection{Results and Discussion}

Professional teleassistants working in home emergency teleassistance centers and telemedical services for assisting elderly people were predominantly females with an education and training as nurses, medical or telemedical assistants, or office clerks. Their responsibilities include answering the call after an elderly person has pressed the button on an emergency alerting device, to then assess the situation and take measures (around 200 to 500 calls a day, only around $2 \%$ real emergencies), GPS-based locating of elders, or monitoring of health-related devices (e.g., related to blood pressure, weight, respiration). Necessary skills are good local language proficiency (e.g., to understand dialects), friendliness, and computer proficiency.
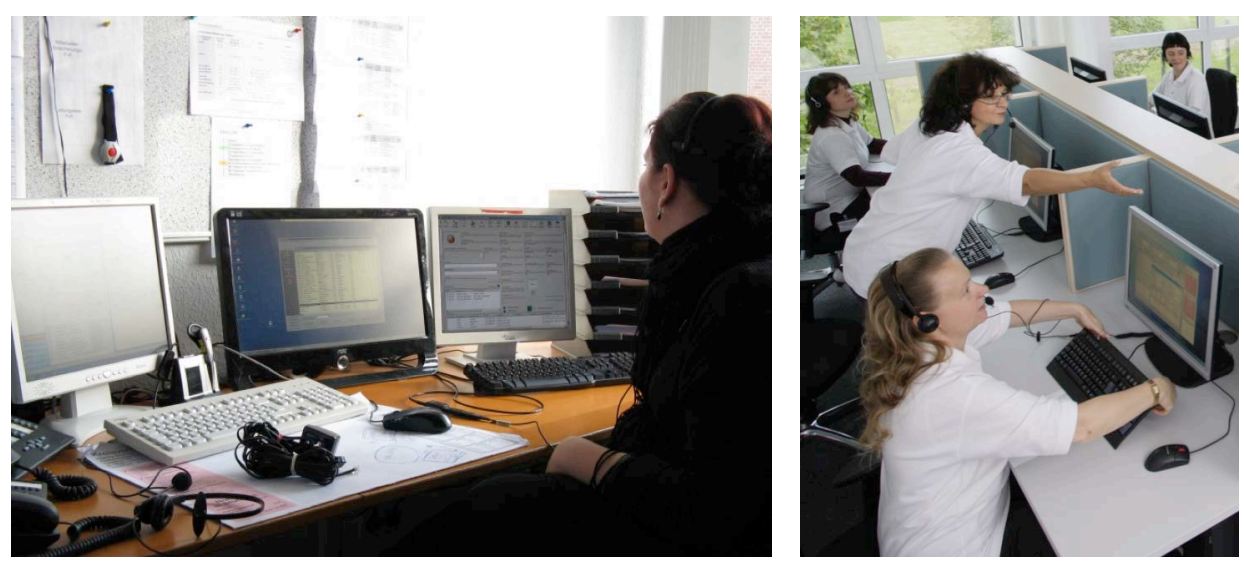

Figure 4. Two contrasting workspaces: large office desk with multi-screen setup as found in the home emergency centers (left); small workspace at the telemedical institution (right)

The work is organized as 24-hour shiftwork. In three of the four institutions visited, people worked in regular offices with large desks with conventional PC's and often multiple screens per person, running various computer programs simultaneously, and multiple telephones or headsets (Figure 4, left). The telemedical institution had smaller workspaces, typical of a call center (Figure 4 , right). We found that teleassistants use a wide variety of computer programs and all had a technology-friendly attitude. Teleassistants also need to know the technical specifics of many different devices in use at the elderly people's homes. Some of the computer programs in use provide them with background information on the caller, e.g., name, age, diseases, medication, phone numbers of relatives, or where to find front door keys. We found such information to also be relevant for the investigated robotic concept and incorporated it in the user interface (see Section 4.7). We also obtained positive initial feedback on the robotic concept and some suggestions. For example, teleassistants stated it would be useful to see the elderly person in case of an emergency (e.g., through the robot's cameras) to better assess the severity of the situation.

We can conclude that the working environment of present teleassistance centers seems suitable for incorporating robotic teleassistance. We further consider it feasible for at least some individuals to handle remote assistance with a robot, perhaps even for more complex tasks like guiding a robotic arm, after receiving training. Their computer, language, and social skills constitute an adequate professional profile. 


\section{Human-Robot Interaction Concept}

In this section, we describe the interaction concept and the user interfaces designed with input from the studies of the preparatory stage.

\subsection{Underlying Concept Decisions and User Interface Requirements}

From the results of the seven preparatory studies (cf. Section 2) and from conceptual goals, we derived design decisions for the human-robot interaction concept and specified 57 requirements (Thayer \& Dorfman, 1990) for its user interfaces as well as requirements for other technical components (e.g., decision making, environment perception). Table 1 lists examples of concept decisions and user interface requirements.

Table 1. Examples of study results and their link to concept decisions and user interface

Study Result
Focus group study (backed by literature): Elderly people
stated they often feel patronized by caregivers and they
long to be independent and self-reliant.

long to be independent and self-reliant.

\section{Concept Decision / User Interface Requirement}

Concept decision: Elderly user shall have as much control over the system as possible

UI requirement: UI for elderly user shall support full control over autonomous services (e.g., start, stop) UI requirement: Elderly user shall be able to choose which remote operator to call for assistance

Focus group study: Informal caregivers were willing to remotely operate a robot and it appealed to them that they could leave the house while knowing they can still help if something should happen to the assisted elderly person.

Interaction analysis: Some required interactions were considered too complex to be handled by untrained users. \& Focus group study: Informal caregivers felt consumed in their care and were afraid of increased burden of reachability with the proposed system (e.g., when on holidays).

Survey on robot service demands: There were substantial differences in user-rated usefulness of robot services (see Section 3.1).

Concept decision: The concept shall include informal caregivers as a user group.

Concept decision: The concept shall include trained professional remote assistants as a user group, available

24 hours a day.

Concept decision: The concept shall focus on userdemanded services as much as possible, considering also other factors (see Section 4.3).

Interaction analysis: It will be important for the elderly user and remote user to communicate during an assistance

UI requirement: The user interfaces shall support audio and video communication between users. about own goals and objects in the apartment).

Ethnography on teleassistance centers: Teleassistants rely on caller information such as medications or the location of door keys.
UI requirement: The user interface for professional users shall have a free text field for writing comments on customers.

\subsection{Robotic Platform}

While we conceive the developed interaction concept as fairly generic, it is initially implemented on the Care-O-bot 3 platform. Care-O-bot 3 (Graf et al., 2009) is the third generation of an assistive service robot for home environments, developed by the Fraunhofer Institute for Manufacturing Engineering and Automation (IPA). The robot (Figure 5) has an omni-directional mobile platform, a manipulator with seven degrees of freedom, a three-finger gripper, a motorized 
sensor head containing a stereo camera and a 3D sensor, and a retractable tray for carrying objects. The robot further features a touch screen integrated in the tray, microphones, speakers, and colored LEDs. A navigation system allows the robot to find its way to locations in the home, avoiding humans and obstacles (Graf, Hans, \& Schraft, 2004). Image processing abilities allow the robot to learn and later recognize and localize objects like bottles or cups (Arbeiter, Fischer, \& Verl, 2010). The robot is further able to detect geometric environment features such as planes in order to identify tables or walls. It can identify grasp configurations and plan arm movements to grasp objects autonomously (Kunz et al., 2010).
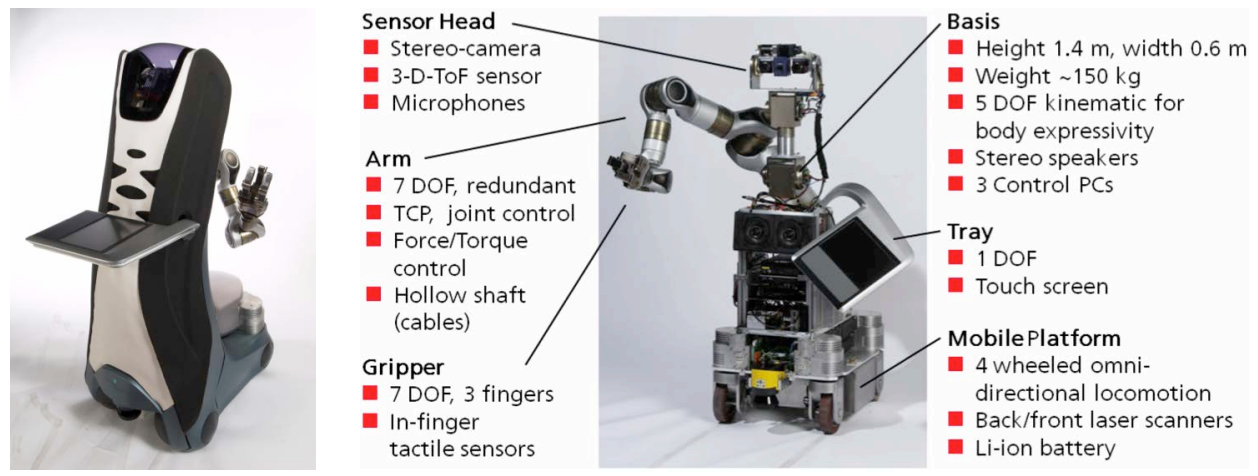

Figure 5. Care-O-bot 3 with and without cover

\subsection{Robot Services}

The concept foresees robotic services that utilize a robot's manipulation capabilities to provide functional assistance. When choosing robot services for implementation on a particular robot, user demand (see Section 3.1) should always be the most important criterion. Further factors to consider are: (a) Necessity of a robot (with manipulation capabilities): For example, reminding people of appointments can also be achieved with much simpler technology. (b) Technological feasibility: For example, as the Care-O-bot 3 platform has only one manipulator, opening packages or food cans is unfeasible. (c) Enabler capability: Certain services can be the foundation of other services. For example, a basic service for manipulation-capable robots is "fetch and carry" (picking objects and placing them elsewhere). This service can enhance emergency services (e.g., robot can bring medication) or make the service of tidying up a room possible.

For each of the robot services listed in Section 3.1, we carried out an assessment considering the factors above and settled to initially focus on the following services for Care-O-bot 3 (cf. Figure 6):

- Fetch and carry and basic manipulation: Grasping objects and delivering them to a different location as well as opening doors or pressing buttons are basic required functions of a manipulating robot and key to achieving a large number of other, more specialized applications.

- Emergency assistance: The elderly person places an emergency call (e.g., in case of a fall). A remote operator moves the robot to the emergency location and assesses the health status or type of injury through the robot's cameras. The operator can also use fetch-carry, e.g. to bring medicine, or open the apartment door for the ambulance.

- Difficult objects: For elderly people, objects low on the ground and high up are often difficult to reach. Also, heavy objects can be problematic. Within certain hardware limits, the robot can be used to handle such objects. 

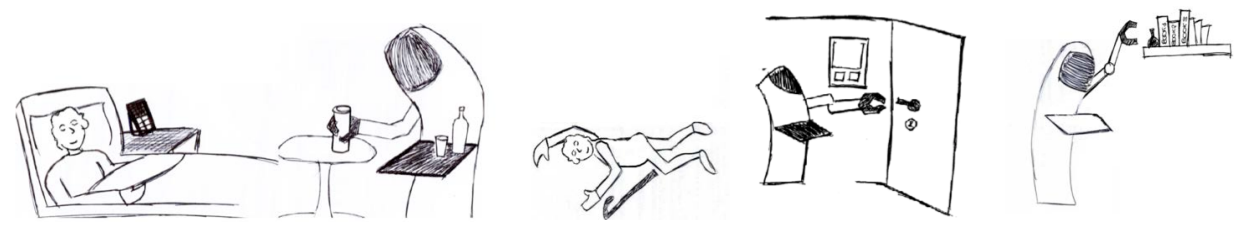

Figure 6. Illustrations of robot services "fetch and carry", "emergency assistance", and "difficult objects"

\subsection{User Groups, Roles, and Interaction Devices}

As a result of the user studies and the interaction and hardware analyses (see Section 2), the concept foresees three user groups:

- Elderly people living in their original, private home, having difficulties with instrumental activities of daily living or who are prone to falling or the occurrence of other emergency situations

- Informal caregivers (family, friends) caring for an elderly person as specified above and potential caregivers who are willing to provide care but have so far been unable due to geographical distance (in Europe, of $51 \%$ of adults above 70 the nearest child lives more than $1 \mathrm{~km}$ away and of $16 \%$ the nearest child lives more than $25 \mathrm{~km}$ away; Kohli, Künemund, \& Lüdicke, 2005)

- Professional teleassistants, available 24 hours a day in a service center (can be current employees of home teleassistance centers for elderly people having received training for robot remote assistance)

Figure 7 illustrates the roles of users in the interaction concept. Each user group has a different device for interacting with the robot. The devices and their user interfaces scale in their portability and capabilities, from very portable but with only easy-to-use basic functions focusing on autonomous robot services (smartphone-sized touchscreen device for local elderly user, UI-LOC), via still portable and always connected but larger, making some semi-autonomous navigation and grasping functions as well as teleoperated navigation possible (tablet computer for remote caregivers, UI-CG), to desk-based with the widest range of possible interventions including teleoperated grasping ( $\mathrm{PC}$ with 3D interaction devices for professional teleassistants, UI-PRO). The user interfaces of the higher-capability devices also include the functionalities of the lowercapability devices. In addition to the handheld device, a robot's direct interaction capabilities (e.g., speech or gestures) may be used to communicate with the elderly user. For Care-O-bot 3, we chose not to rely on such techniques initially due to reliability concerns.

Autonomous operation is the favored mode, usually initiated by the elderly user. When the system encounters a situation it cannot handle autonomously or if it fails to execute an action, a human remote operator is contacted following a call priority chain. Informal caregivers are proposed first because their assistance is free of charge. If they are unavailable, if the request cannot be handled with their device, or if the elderly user chooses so, the request is forwarded to the 24-hour teleassistance center. Remote calls and assistance requests can also be initiated manually by all users. For example, the elderly user might place a request if a required autonomous function is not present in the user interface or a required object is not in the database and needs to be taught. 


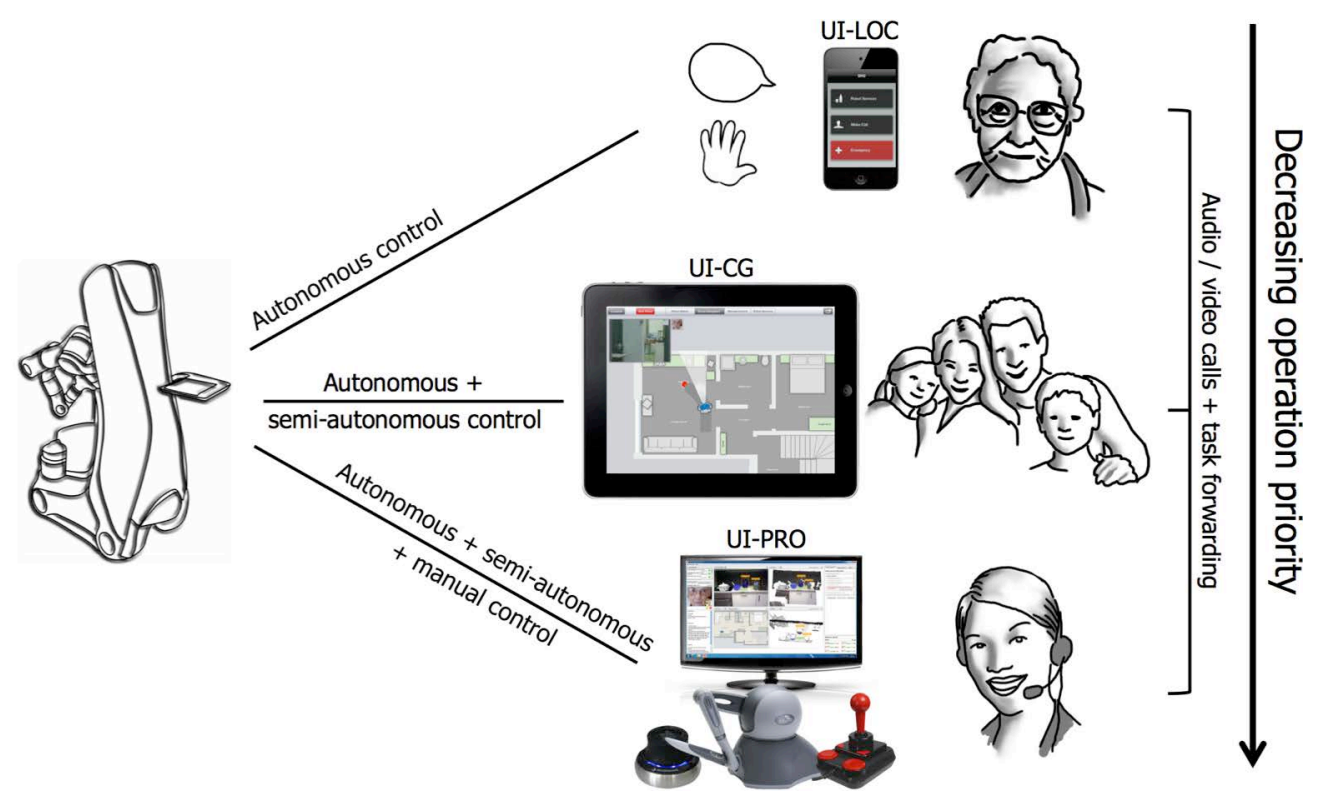

Figure 7. Roles of users and interaction devices in the interaction concept

The prospect of using manipulation-capable robots in the homes of elderly people with remote operators intervening is associated with many ethical concerns (cf., Sharkey \& Sharkey, 2012) about such themes as loss of control, liberty, and privacy of the elderly people (e.g., when a remote operator uses the robot without consent), safety and responsibility in case of damages to people or property, or unreasonable increase of caregiver burden due to exploiting usage behavior of elderly people. While it is out of scope to discuss these concerns exhaustively in the present article, our basic approach to ensure consented usage has been a two-step process occurring before each remote control session: First, a conversation between users is established in the form of an audio or video call. The communication is important for the elderly person to get acquainted with who is going to intervene in the home. Also, it is important throughout the subsequent remote control session, to increase trust and for the remote operator to be able to ask questions, e.g. about the elderly user's goals or the location of objects to be fetched. In the second step, before remote control can take place, the elderly user has to explicitly authorize it by pressing a button. The only exception to this rule is emergency calls where control is granted immediately. It could be considered to relax this authorization principle to the benefit of elderly people with severe handicaps, health-related risks, or cognitive impairments but this would require a thorough investigation of ethical tradeoffs such as privacy and liberty loss beforehand.

The concept and user interfaces leave room for incorporating learning and teaching functions, intended mainly for UI-CG and UI-PRO. For Care-O-bot, UI-CG and UI-PRO incorporate functions for teaching objects and action sequences. If multiple users can teach new skills or the robot learns by itself, it is important to communicate newly acquired skills to the other users. Services that presently cannot be provided autonomously with sufficient reliability may have to be provided exclusively through remote operation. With the progression of technology and increasing use of machine learning and teaching techniques, robots employing the interaction concept should become more and more autonomous over time, gradually reducing the involvement of human remote operators.

The following three sections describe the user interfaces for the three user groups. We created horizontal prototypes, i.e., realistic interactive showcases of how the final product would look like and behave, but with restricted functionality and without a real ability to control the robot. Videos taken with the robot's RGB and 3D cameras were employed to simulate robot navigation and 
manipulation. The user interface prototypes were tested in a usability study (see Section 2). The subsequently presented user interfaces represent the second iteration, improved based on the study results.

\subsection{User Interface for Local Elderly User (UI-LOC)}

The user interface for elderly people focuses on autonomous robot functions. The user selects a service such as fetching an object or opening a door and the robot navigates and manipulates autonomously. If a problem occurs, a remote operator is called for help. As a consequence of the usability study, some of our design goals for this iteration were to use few hierarchical menu levels, a step-by-step interaction rationale with only currently relevant information on the screen, large buttons and text, and no foreign or technology-oriented language.

The main menu (Figure 8, screen 1) has three large buttons: "Robot Services" contains the autonomous services such as fetching objects or opening doors. "Make Call" provides the calling functionality to informal caregivers and the 24-hour service and "Emergency" the emergency assistance functionality where a professional operator is called who can assess the health status through the robot's cameras and take further measures. Screen 2 in Figure 8 appears after tapping on "Robot Services" in the main menu. Robot services may include variables such as objects to fetch and destinations to deliver them (screen 3). The fourth and fifth screens show further steps of the "Bring objects" service. In the last screen, an example is provided of how an elderly user by simple means can assist the robot's object detection in case of a failure. In this case the bottle to be fetched is at its expected location but surrounded by other objects. Therefore, detection failed. The user was asked before screen 6 if he or she can see the bottle in the picture provided by the robot's camera. Had the local user chosen "no", then the robot would have searched at a different location or a remote user would have been called.

\subsection{User Interface for Remote Caregivers (UI-CG)}

In addition to the functionalities of UI-LOC, the user interface for caregivers, UI-CG, offers semiautonomous navigation, scene-based autonomous manipulation without pre-defined action sequences, object teaching, and action sequence editing. We currently consider it too challenging to also implement low-level or semi-autonomous manipulation on a tablet computer.

Screen 1 in Figure 9 shows the room plan-based navigation, which is the primary navigation mode although fine adjustments and base rotation can be performed with manual controls in the scene-based view (screen 2). In the room plan, the user can tap on surfaces (highlighted in green) to send the robot there or to deliver objects. The user can also tap on any other point in the room plan to specify it as a navigation destination. The robot indicates the planned navigation path and updates it while moving. The path can be dragged by the user to make adjustments. Sensor data can be overlaid on the room plan, for example for the user to identify obstacles. The stylized beam of light indicates the robot's current field of view as displayed in the video scene in the upper left.

Screen 2 shows the scene-based interaction mode. Recognized surfaces like tables and objects, door handles, or buttons of an appliance are augmented onto the scene. In screen 2, the user has tapped on the water bottle and is presented with the options of placing it on the tray or tidying it away. The user has the option to teach an object via rotation on the gripper (requires local user involvement) or by assisting a generic object recognition algorithm by encircling an object of interest. A name and a category can then be assigned to the object. Screen 3 shows the robot's current status. Newly learned objects and action sequences are displayed so any user can see what other users have taught in his absence. The user interface for creating and changing action sequences is shown in screen 4 . There are actions (e.g., bring, open, tidy up) and items sorted by category (e.g., surfaces, movable objects, rooms). Sequences can be generated by consecutively dragging and dropping action elements and item elements onto a timeline. 
Mast et al., Remote Interaction Concept for Assistive Robots

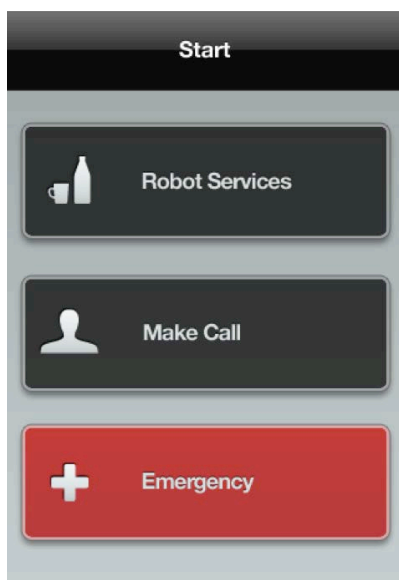

1. Main menu

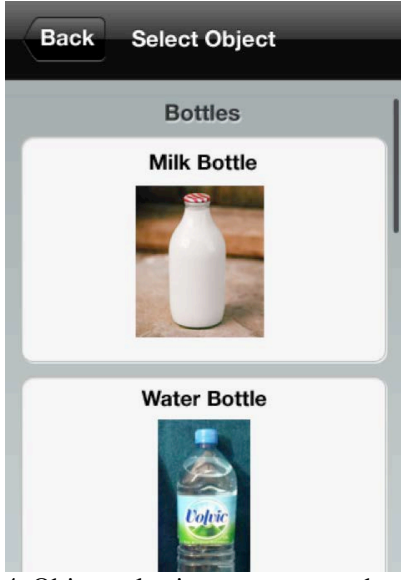

4. Object selection screen, sorted by category, with pictures taken during object learning

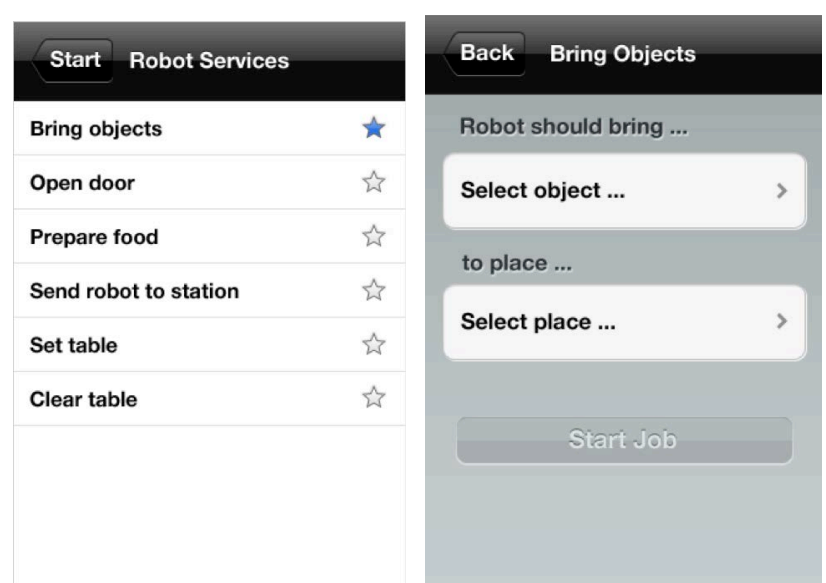

2. Robot services with favorite service on the top (blue star)

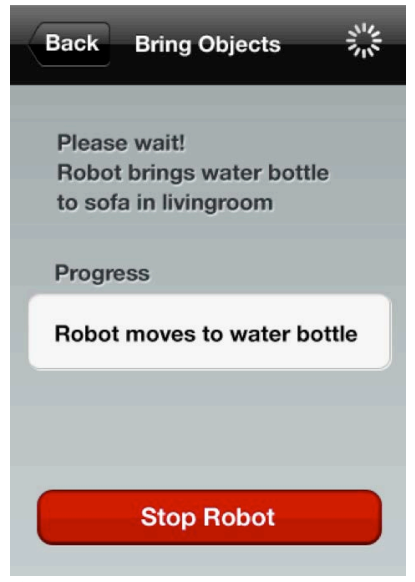

5. Robot service progress screen with option to interrupt the task
3. "Bring objects" function, main screen

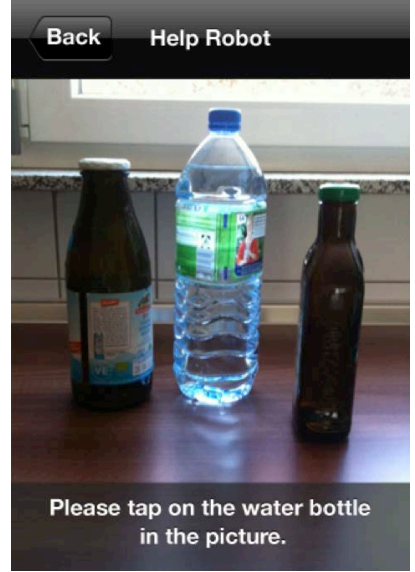

6. User can provide basic help by narrowing down search space in case of an object detection error

Figure 8. Screens from user interface for local elderly user (UI-LOC)

An extension to the room plan-based navigation is under development, incorporating a 3D model of the apartment combined with 3D sensor data and exocentric views to better assess the local situation. Also, since the limited field of view of RGB cameras is problematic for orientation, we consider enhancing the scene view by surrounding it with 3D data or additional RGB data (cf. Michaud et al., 2010; Lazewatsky \& Smart, 2011).

\subsection{User Interface for Professional Teleassistants (UI-PRO)}

The 24-hour professional teleassistant user interface, UI-PRO, as the last instance that can solve problems with the robot, offers several advanced functions. Foremost, it provides semiautonomous telemanipulation. Figure 10 shows the workstation hardware concept. The operator uses a personal computer with a large display. Optionally, a 3D display could be used to better assess depth in a scene. The perspective of 3D scenes can be adjusted with a 3D mouse (e.g., 3Dconnexion SpaceNavigator). Since robots differ in their manipulation hardware (e.g., one versus two arms, different degrees of freedom, different gripper dexterities), there is no single ideal telemanipulation device. We consider high-precision stylus-based 3D manipulation devices a 


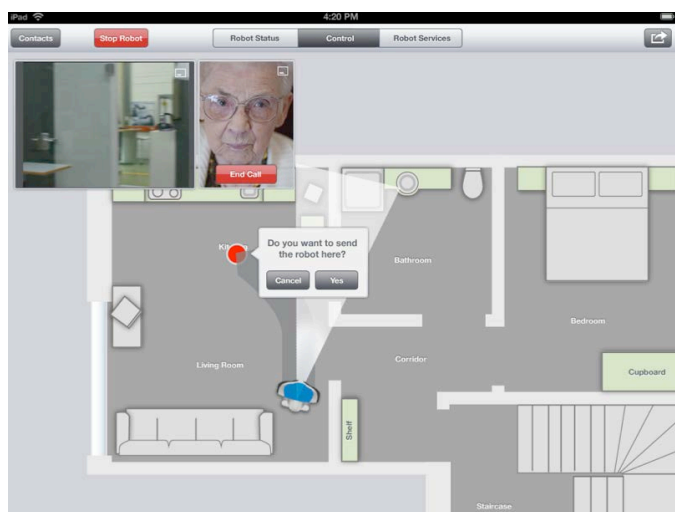

1. Room plan view with robot about to move from living room to kitchen (robot camera view and video communication in upper left)

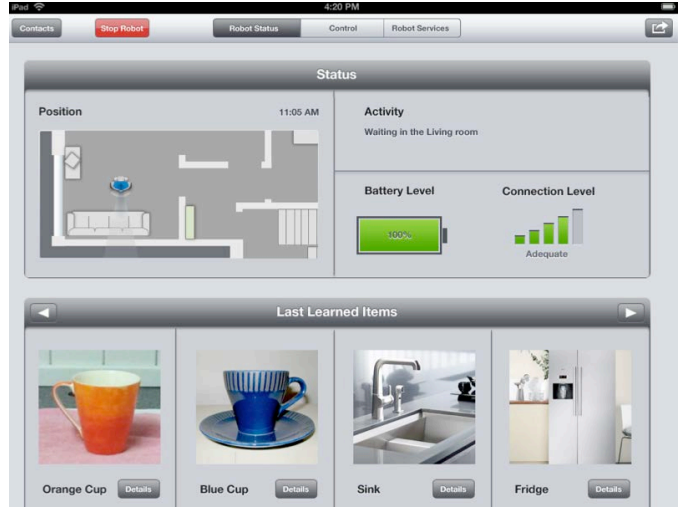

3. Status screen with last learned items at the bottom

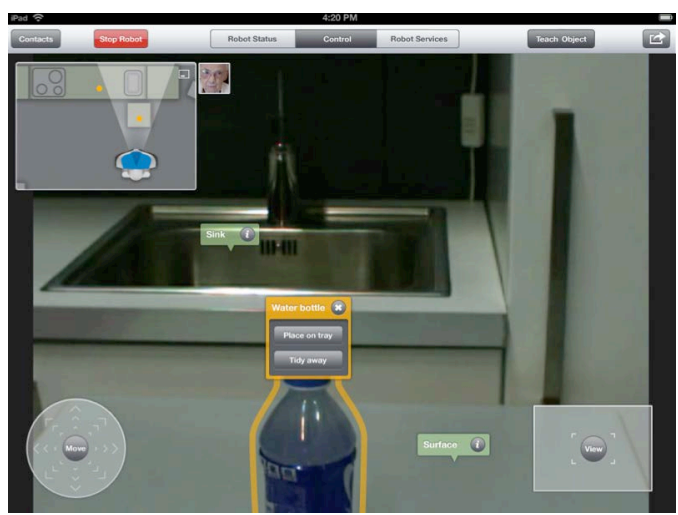

2. Scene view with robot having arrived in kitchen with highlighted surfaces and furniture (light green) and recognized object for manipulation (yellow)

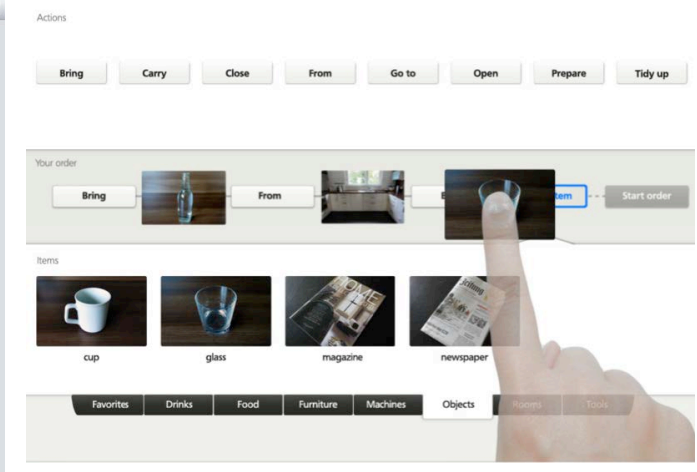

4. Drag-and-drop-based action sequence editing

Figure 9. Screens from user interface for informal caregivers (UI-CG)

reasonable choice for one-armed robots like Care-O-bot, offering intuitive interaction (e.g., Sensable Phantom). A conventional joystick is used for manual navigation and the workstation further contains two emergency stop buttons.

Figure 11 shows the main screen of the UI-PRO user interface. All necessary functions for the remote operator's central tasks have been integrated into one window. The user interface follows the rationale of the left pane being for customer-related functions, the center for the views of the apartment, and the right pane for robot-related functions. In the left pane, the list of incoming calls appears at the top (emergency calls are highlighted and prioritized), each call indicating the problem that occurred with the robot. The middle of the left panel shows active calls. Simultaneous sessions are possible, as the operator may have to wait for a robot to finish a task. At the bottom is a free text field for viewing and entering information about customers such as required medicine or the location of house keys for cases of emergency, as found in current systems in use at teleassistance centers (cf. Section 3.2). The operator can also switch to another tab for customer management and call history.

The right pane contains three tabs. "Current sequence" shows the action sequence step where the robot encountered an error (red) and the post-state that has to be reached by the human operator in order to resume autonomous operation (e.g., if the robot failed to bring he manipulator to the pre-grasp position, reaching the pre-grasp position becomes the user's goal). The tab "Robot services" contains the autonomous functions of UI-LOC as well as the action sequences editing function of UI-CG. The "Objects" tab contains the object database and object teaching functions. 


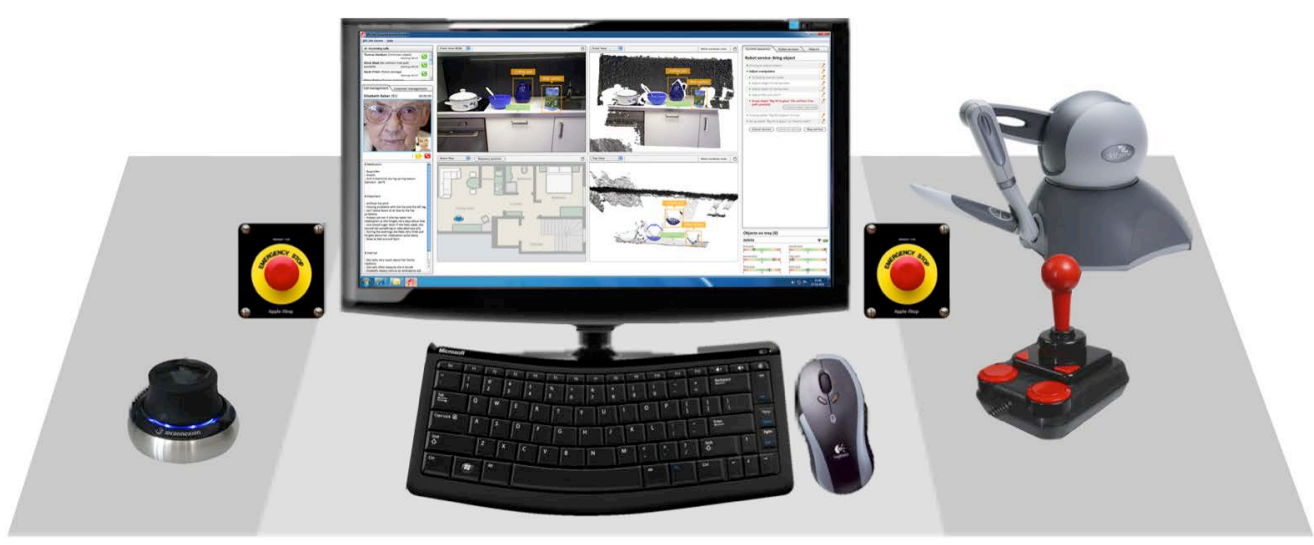

Figure 10. Workstation hardware concept for 24-hour teleassistance service staff consisting of monitor, keyboard, mouse, two emergency stop buttons, joystick, 3Dconnexion SpaceNavigator 3D mouse (front left), and Sensable Phantom stylus-based 3D haptic manipulation device (back right)

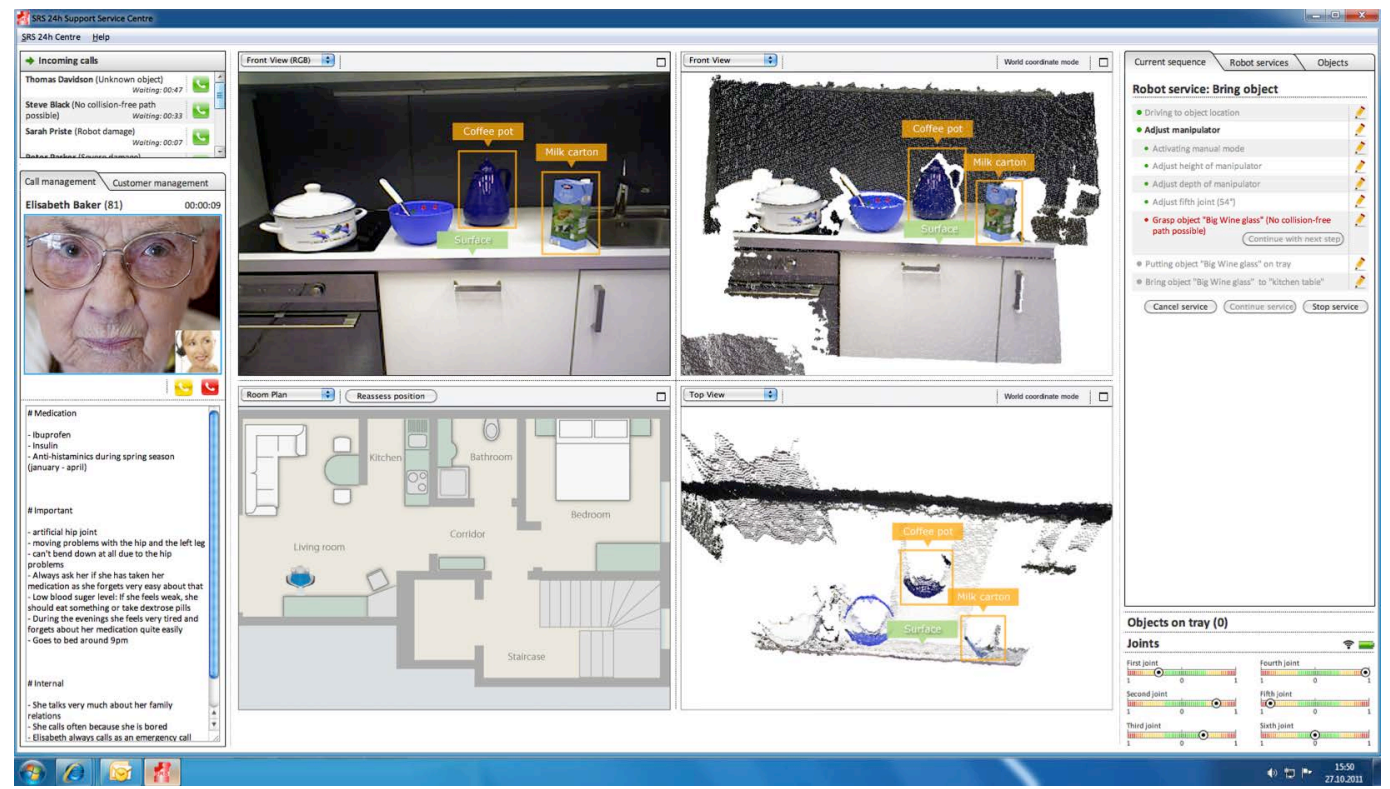

Figure 11. User interface for professional teleassistants (UI-PRO)

Advanced object teaching functions are offered, based on placing and scaling a generic 3D model in the scene to teach object boundaries and grasping points. At the bottom of the right tab, objects on the tray and the arm's joint limits are indicated.

Most screen space is allocated to the center area. In each of the four windows, the operator can choose between the robot's RGB video camera, several point-cloud-based fixed-perspective views (front, top, left, right, virtual gripper camera), 3D room model-based views and the 2D room plan (with ability to adjust navigation trajectories). Because 3D cameras only capture a small part of a scene, point cloud-based views make use of historic data with an option to refresh the point cloud data by means of the robot rotating 360 degrees at its current position. A further mode combines point cloud data (with the advantage of being up-to-date) with room model data (with the 
advantage of being well comprehensible). Each of the four windows can be maximized. The operator can use the 3D mouse to adjust views (e.g., rotate, zoom) and the stylus device for manipulation. For semi-autonomous manipulation, the operator first moves the stylus to the grasping target in a wireframe-based simulation mode. When confident with the result in simulation, the operator executes the movement. Trajectories are then planned and executed by the robot. This semi-autonomous mode is not subject to network delays. The coordinate mode for using the stylus device can be switched between world coordinate mode and tool coordinate mode.

\section{Conclusion}

The work presented in this article encompasses the design of a human-robot interaction concept for service robots to enable functional assistance to elderly people. The user interfaces along with the underlying technologies for environment modeling, robot control, machine learning, and decision-making are currently under development, where the iterative process with continuous user involvement is sustained. Further studies on usability, user acceptance, as well as on ethical issues and on cost-effectiveness are underway. It is in the nature of open-ended creative work that further refinements will be made along the way, when additional feedback has been gathered from users. We see the work presented as a vision for allowing elderly people to receive functional support in the near term, while fully autonomous robots are not yet ready for the market. While we have focused on elderly people, aspects of the concept may also apply to service robots with other target groups, for example robots to assist handicapped users or general-purpose domestic robots. When the technological state of the art has progressed, tasks carried out by remote human operators today could gradually be replaced with autonomous behavior and local interaction. Ideally, the proposed interaction concept would become obsolete one day, when robots can reliably handle all situations autonomously.

\section{Acknowledgement}

The work presented in this article was carried out as part of the research project "Multi-Role Shadow Robotic System for Independent Living" (SRS), funded by the European Commission under the 7th Framework Programme (grant agreement no. 247772).

\section{References}

Arbeiter, G., Bubeck, A., Fischer, J., \& Graf, B. (2009). Teilautonome mobile Roboter zur Fernwartung prozesstechnischer Anlagen. In: K. Bender et al. (Eds.), Mesago: SPS/IPC/DRIVES 2009. Tagungsband (pp. 173-181). Nürnberg: VDE-Verlag.

Arbeiter, G., Fischer, J., \& Verl, A. (2010). 3D perception and modeling for manipulation on Care-O-bot 3. In: Conference Digest of IEEE/RAS International Conference on Robotics and Automation (ICRA), Anchorage, Alaska, USA (pp. XXIII-XXVII). Piscataway, NJ, USA: IEEE Press.

Bayer, A., \& Harper, L. (2000). Fixing to stay. A national survey of housing and home modification issues. Washington, DC: AARP. Retrieved from: http://assets.aarp.org/ rgcenter/il/home_mod.pdf

Becker, S., Böhm, U., Röhrig, A., Stuhler, H., \& Wurm, S. (2007). Tätigkeiten in Haushalt, Freizeit und Ehrenamt. In W. Friesdorf, \& A. Heine (Eds.), sentha - seniorengerechte Technik im häuslichen Alltag (pp. 57-68). Berlin: Springer.

Beer, J. M., \& Takayama, L. (2011). Mobile remote presence systems for older adults: Acceptance, benefits, and concerns. Proceedings of HRI, Lausanne, Switzerland, 19-26. doi: $10.1145 / 1957656.1957665$ 
Beyer, H. \& Holtzblatt, K. (1998). Contextual design. Defining customer-centered systems. San Francisco: Morgan Kaufmann.

Bohren, J., Rusu, R. B., Jones, E. G., Marder-Eppstein, E., Pantofaru, C., Wise, M., ... Holzer, S. (2011). Towards autonomous robotic butlers: Lessons learned with the PR2. Proceedings of IEEE International Conference on Robotics and Automation (ICRA), Shanghai, China, 55685575. doi:10.1109/ICRA.2011.5980058

Boissy, P., Corriveau, H., Michaud, F., Labonté, D., \& Royer, M. (2007). A qualitative study of in-home robotic telepresence for home care of community-living elderly subjects. Journal of Telemedicine and Telecare, 13, 79-84. doi:10.1258/ 135763307780096195

Bruemmer, D. J., Dudenhoeffer, D. D., \& Marble, J. L. (2002). Dynamic-autonomy for urban search and rescue. AAAI Technical Report WS-02-18. Retrieved from: http://www .aaai.org/Papers/Workshops/2002/WS-02-18/WS02-18-006.pdf

Buss, M., Peer, A., Schauß, T., Stefanov, N., Unterhinninghofen, U., Behrendt, S., ... Sarkis, M. (2010). Development of a multi-modal multi-user telepresence and teleaction system. International Journal of Robotics Research, 29, 1298-1316. doi:10.1177/0278364909351756

Cooper, A. (1999). The inmates are running the asylum. Why high tech products drive us crazy and how to restore the sanity. Indianapolis: Macmillan Publishing.

Crandall, J. W., \& Goodrich, M. A. (2001). Experiments in adjustable autonomy. Proceedings of IEEE International Conference on Systems, Man, and Cybernetics, 1624-1629. doi: 10.1109/ICSMC.2001.973517

Dautenhahn, K., Woods, S., Kaouri, C., Walters, M. L., Koay, K. L. , \& Werry, I. (2005). What is a robot companion - friend, assistant or butler?. Proceedings of IEEE/RSJ International Conference on Intelligent Robots and Systems (IROS), Edmonton, Canada, 1192-1197. doi:10.1109/IROS.2005.1545189

Davis, F. D. (1989). Perceived usefulness, perceived ease of use, and user acceptance of information technology. MIS Quarterly, 13, 319-340.

Deegan, P., Grupen, R., Hanson, A., Horrell, E., Ou, S., Riseman, E., ... Xie, D. (2008). Mobile manipulators for assisted living in residential settings. Autonomous Robots, 24, 179-192. doi:10.1007/s10514-007-9061-8

Dorais, G. A., Bonasso, R. P., Kortenkamp, D., Pell, B., \& Schreckenghost, D. (1998). Adjustable autonomy for human-centered autonomous systems on mars. Proceedings of International Conference of the Mars Society.

Dumas, J. C., \& Fox, J. E. (2008). Usability testing: Current practice and future directions. In A. Sears \& J. A. Jacko (Eds.), The handbook of human-computer interaction (pp. 1129-1150). New York: Lawrence Erlbaum Associates.

Endsley, M. R. (1988). Design and evaluation for situation awareness enhancement. Proceedings of the Human Factors Society 32nd Annual Meeting, Santa Monica, CA, 97-108.

Ezer, N., Fisk, A. D., Rogers, W.A. (2009). More than a servant: Self-reported willingness of younger and older adults to having a robot perform interactive and critical tasks in the home. Proceedings of the Human Factors and Ergonomics Society 53rd Annual Meeting, 136-140. doi:10.1518/107118109X12524441079382

Faucounau, V., Wu, Y., Boulay, M., Maestrutti, M., Rigaud, A., \& the QuoVADis project (2009). Caregivers' requirements for in-home robotic agent for supporting community-living elderly subjects with cognitive impairment. Technology and Health Care, 17, 33-40. doi:10.3233/THC-2009-0537 
Mast et al., Remote Interaction Concept for Assistive Robots

Ferland, F., Pomerleau, F., Le Dinh, C. T., \& Michaud, F. (2009). Egocentric and exocentric teleoperation interface using real-time, 3d video projection. Proceedings of HRI, 37-44. doi:10.1145/1514095.1514105

Gonzalez, M. F., Facal, D., Navarro, A. B., Geven, A., \& Tscheligi, M. (2011). Analysis of older users' perceived requests and opportunities with technologies: A scenario-based assessment. International Journal of Ambient Computing and Intelligence, 3(1), 42-52. doi:10.4018/jaci.2011010105

Goodrich, M. \& Schultz, A. C. (2007). Human-robot interaction: A survey. In B. Bederson et al. (Eds.), Foundations and trends in human-computer interaction, Vol. 1 (pp. 203-275). Hanover, MA, USA: now Publishers. doi:10.1561/1100000005

Graf, B., Hans, M., \& Schraft, R. D. (2004). Mobile robot assistants - Issues for dependable operation in direct cooperation with humans. IEEE Robotics \& Automation Magazine, 11(2), 67-77. doi:10.1109/MRA.2004.1310943

Graf, B., Parlitz, C., \& Hägele, M. (2009). Robotic home assistant Care-O-bot 3 product vision and innovation platform. In: J. A. Jacko (Ed.), Human-computer interaction, part II, HCI International 2009, San Diego, CA, USA, Lecture Notes in Computer Science, Vol. 5611 (pp. 312-320). Berlin: Springer. doi: 10.1007/978-3-642-02577-8_34

Harmo, P., Taipalus, T., Knuuttila, J., Vallet, J., \& Halme, A. (2005). Needs and solutions - home automation and service robots for the elderly and disabled. Proceedings of IEEE/RSJ International Conference on Intelligent Robots and Systems (IROS), Edmonton, Canada, 32013206. doi: 10.1109/IROS.2005.1545387

Hassenzahl, M., Burmester, M., \& Koller, F. (2003). AttrakDiff: Ein Fragebogen zur Messung wahrgenommener hedonischer und pragmatischer Qualität. In: J. Ziegler \& G. Szwillus (Eds.), Mensch \& Computer 2003. Interaktion in Bewegung (pp. 187-196). Stuttgart: Teubner.

Hibbert, D., Mair, F. S., May, C. R., Boland, A., O’Connor, J., Capewell, S., \& Angus, R. M. (2004). Health professionals' responses to the introduction of a home telehealth service. Journal of Telemedicine and Telecare, 10, 226-230. doi:10.1258/1357633041424386

ISO 9241-210 (2010). Ergonomics of human-system interaction - part 210: Human-centred design for interactive systems. International Standardization Organization.

Iwata, H., \& Sugano, S. (2009). Design of human symbiotic robot TWENDY-ONE. Proceedings of IEEE International Conference on Robotics and Automation (ICRA), Kobe, Japan, 580-586. doi: 10.1109/ROBOT.2009.5152702

Kemp, C. C., Edsinger, A., \& Torres-Jara, E. (2007). Challenges for robot manipulation in human environments. IEEE Robotics \& Automation Magazine, 14(1), 20-29. doi: 10.1109/MRA.2007.339604

Khan, Z. (1998). Attitudes towards intelligent service robots (Report No. TRITA-NA-P9821, IPLab-154). Stockholm: Royal Institute of Technology.

Kohli, M., Künemund, H., \& Lüdicke, J. (2005). Family structure, proximity and contact. In Börsch-Supan et al. (Eds.), Health, ageing and retirement in Europe (pp. 164-170). Mannheim, Germany: Mannheim Research Institute for the Economics of Aging (MEA).

Kunz, T., Reiser, U., Stilman, M., \& Verl, A. (2010). Real-time path planning for a robot arm in changing environments. Proceedings of IEEE/RSJ International Conference on Robots and Intelligent Systems (IROS), Taipei, Taiwan, 5906-5911. doi:10.1109/IROS.2010.5653275 
Labonté, D., Boissy, P., \& Michaud, F. (2010). Comparative analysis of 3-D robot teleoperation interfaces with novice users. IEEE Transactions on Systems, Man, and Cybernetics, 40, 13311342. doi: 10.1109/TSMCB.2009.2038357

Lazewatsky, D. A., \& Smart, W. D. (2011). A panorama interface for telepresence robots. Proceedings of HRI, 177-178. doi:10.1145/1957656.1957719

Martens, C., Prenzel, O., \& Gräser, A. (2007). The rehabilitation robots FRIEND-I \& II: Daily life independency through semi-autonomous task-execution. In S. S. Kummo (Ed.), Rehabilitation robotics (pp. 137-162).

Mason, M., \& Lopes, M. (2011). Robot self-initiative and personalization by learning through repeated interactions. Proceedings of HRI, 433-440. doi:10.1145/1957656. 1957814

Mast, M., Burmester, M., Berner, E., Facal, D., Pigini, L., \& Blasi, L. (2010). Semi-autonomous teleoperated learning in-home service robots for elderly care: A qualitative study on needs and perceptions of elderly people, family caregivers, and professional caregivers. Proceedings of the 20th International Conference on Robotics and Mechatronics, Varna, Bulgaria, 1-6.

McDowell, I. (2006). Measuring health. A guide to rating scales and questionnaires (3rd ed.). New York: Oxford University Press.

Merton, R. K., Fiske, M., \& Kendall, P. I. (1990). The focused interview. A manual of problems and procedures (2nd ed.). New York: The Free Press.

Meyer, S. (2011). Mein Freund der Roboter. Servicerobotik für ältere Menschen - eine Antwort auf den demografischen Wandel? Berlin: VDE-Verlag.

Michaud, F., Boissy, P., Labonté, D., Brière, S., Perreault, K., Corriveau, H., ... Létourneau, D. (2010). Exploratory design and evaluation of a homecare teleassistive mobile robotic system. Mechatronics, 20, 751-766. doi:10.1016/j.mechatronics.2010.01.010

Milligan, C., Roberts, C., \& Mort, M. (2011). Telecare and older people: Who cares where? Social Science \& Medicine, 72, 347-354. doi:10.1016/j.socscimed.2010.08.014

Mukai, T., Hirano, S., Nakashima, H., Kato, Y., Sakaida, Y., Guo, S., \& Hosoe, S. (2010). Development of a nursing-care assistant robot RIBA that can lift a human in its arms. Proceedings of IEEE/RSJ International Conference on Robots and Intelligent Systems (IROS), Taipei, Taiwan, 5996-6001. doi:10.1109/IROS.2010.5651735

Nehaniv, C. L., \& Dautenhahn, K. (Eds.). (2007). Imitation and social learning in robots, humans and animals. Behavioural, social and communicative dimensions. Cambridge, UK: Cambridge University Press.

Nielsen, C. W., Goodrich, M. A., \& Ricks, R. W. (2007). Ecological interfaces for improving mobile robot teleoperation. IEEE Transactions on Robotics, 23, 927-941. doi:10.1109/TRO.2007.907479

O'Malley, M. K., \& Ambrose, R. O. (2003). Haptic feedback applications for Robonaut. Industrial Robot, 30, 531-542. doi:10.1108/01439910310506800

Pollack, M. E., Brown, L., Colbry, D., Orosz, C., Peintner, B., Ramakrshnan, S., ... Roy, N. (2002). Pearl: A mobile robotic assistant for the elderly. Papers from the AAAI Workshop on Automation as Caregiver (Technical Report WS-02-02). Retrieved from: http://www.aaai.org/Papers/Workshops/2002/WS-02-02/WS02-02-013.pdf

Ray, C., Mondada, F., \& Siegwart, R. (2008). What do people expect from robots? Proceedings of IEEE/RSJ International Conference on Intelligent Robots and Systems (IROS), Nice, France, 3816-3821. doi:10.1109/IROS .2008.4650714 
Rosson, M. B., \& Carroll, J. M. (2008). Scenario-based design. In: A. Sears \& J. A. Jacko (Eds.), The human-computer interaction handbook (2nd ed., pp. 1041-1060). New York: Lawrence Erlbaum Associates.

Sharkey, A., \& Sharkey, N. (2012). Granny and the robots: ethical issues in robot care for the elderly. Ethics and Information Technology, 14, 27-40. doi:10.1007/s10676-010-9234-6

Schermerhorn, P., \& Scheutz, M. (2009). Dynamic robot autonomy: Investigating the effects of robot decision-making in a human-robot team task. Proceedings of ICMI-MLMI, Cambridge, MA, USA, 63-70. doi: 10.1145/1647314.1647328

Sheridan, T. B. (1992). Telerobotics, automation, and human supervisory control. Cambridge, MA, USA: MIT Press.

Shiomi, M., Sakamoto, D., Kanda, T., Ishi, C. T., Ishiguro, H., \& Hagita, N. (2008). A semiautonomous communication robot - a field trial at a train station. Proceedings of HRI, 303-310. doi:10.1145/1349822.1349862

Song, T. H., Park, J. H., Chung, S. M., Hong, S. H., Kwon, K. H., Lee, S., \& Jeon, J. W. (2007). A Study on usability of human-robot interaction using a mobile computer and a human interface device. Proceedings of Mobile HCI, 462-466. doi:10.1145/1377999.1378055

Sung, J., Christensen, H. I., \& Grinter, R. E. (2009). Sketching the future: Assessing user needs for domestic robots. Proceedings of IEEE International Symposium on Robot and Human Interactive Communication (RO-MAN), Toyama, Japan, 153-158. doi: 10.1109/ROMAN.2009.5326289

Thayer, R. H., \& Dorfman, M. (Eds.). (1990). System and software requirements engineering. Los Alamitos, CA, USA: IEEE Computer Society Press.

Thomaz, A. L., \& Breazeal, C. (2008). Teachable robots: Understanding human teaching behavior to build more effective robot learners. Artificial Intelligence, 172, 716-737. doi:10.1016/j.artint.2007.09.009

United Nations (2010). World Population Ageing 2009. New York: United Nations. Retrieved from: http://www.un.org/esa/population/

Wada, K., Shibata, T., Asada, T., \& Musha, T. (2007). Robot therapy for prevention of dementia at Home - results of preliminary experiment. Journal of Robotics and Mechatronics, 19, 691697.

Weiss, A., Wurhofer, D., Buchner, R., Tscheligi, M., \& Blasi, L. (2009). Development of a teleoperator interface for humanoid robots by means of heuristic evaluation technique. Proceedings of Towards Autonomous Robotic Systems (TAROS), 236-241.

Zamora, G., Etxeberria, I., Ansorena, X., García, A., Pigini, L., Facal, D., \& Urdaneta, E. (2011). "The house looks messy, but it's easier for me" - Applied ethnography, domestic robotic solutions and elderly people. ESA 10th Conference. Abstract Book, 36-37.

Authors' contact information: M. Mast, mast@hdm-stuttgart.de; M. Burmester, burmester@hdmstuttgart.de; K. Krüger, kruegerk@hdm-stuttgart.de; S. Fatikow, sf043@hdm-stuttgart.de; G. Arbeiter, georg.arbeiter@ipa.fraunhofer.de; B. Graf, birgit.graf@ipa.fraunhofer.de; G. Kronreif, gernot.kronreif@acmit.at; L. Pigini, lpigini@dongnocchi.it; D. Facal, david.facal@ingema.es; R. Qiu, qiur@cardiff.ac.uk 